\title{
Elektrokonvulsionstherapie im Kindes- und Jugendalter
}

\section{Eine retrospektive Erhebung an drei universitären Zentren bei 12- bis 17-jährigen Patient_innen}

\author{
Isabel Methfessel ${ }^{1}$, Steffen Weirich², Boris Rothermel ${ }^{3}$, Jesse Crozier ${ }^{4}$, Matthias Besse ${ }^{1}$, \\ Martin Reinhardt², Johannes Buchmann², Alexander Dück², David Zilles-Wegner ${ }^{1}$, \\ Frank Häßler ${ }^{5}$, Michael Kölch², Henrik Uebel von Sandersleben ${ }^{4}$, Luise Poustka ${ }^{4}$ und \\ Alexander Sartorius 6
}

Klinik für Psychiatrie und Psychotherapie, Universitätsmedizin Göttingen, Göttingen

Klinik für Psychiatrie, Neurologie, Psychosomatik und Psychotherapie im Kindes- und Jugendalter, Universitätsmedizin Rostock, Rostock

Klinik für Psychiatrie und Psychotherapie des Kindes- und Jugendalters, Zentralinstitut für Seelische Gesundheit,

Medizinische Fakultät Mannheim/Universität Heidelberg, Mannheim

4 Klinik für Kinder- und Jugend psychiatrie/Psychotherapie, Universitätsmedizin Göttingen, Göttingen

5 Tagesklinik für Kinder- und Jugendpsychiatrie, GGP, Gruppe Rostock, Rostock

6 Klinik für Psychiatrie und Psychotherapie, Zentralinstitut für Seelische Gesundheit, Medizinische Fakultät Mannheim/ Universität Heidelberg, Mannheim

\begin{abstract}
Zusammenfassung: Hintergrund und Zielsetzung: Die Elektrokonvulsionstherapie (EKT) ist ein hoch wirksames und in der Erwachsenenpsychiatrie gut untersuchtes Therapieverfahren, das gerade bei schwerwiegenden depressiven und psychotischen Erkrankungen erfolgreich eingesetzt wird. Obwohl schwere Krankheitsverläufe auch im kinder- und jugend psychiatrischen Bereich vorkommen, erfolgt ein Einsatz der EKT hier deutlich seltener. Dies liegt womöglich auch daran, dass kaum systematisch erhobene Daten zur Anwendung, Wirksamkeit und Verträglichkeit der EKT bei kinder- und jugendpsychiatrischen Patient_innen existieren. Ziel dieser Arbeit ist die Untersuchung und Darstellung von Krankheitsverläufen unter EKT-Behandlung im kinder- und jugendpsychiatrischen Bereich und damit eine Vergrößerung der bestehenden Datenbasis zu diesem Thema. Methoden: Es erfolgte eine retrospektive Erhebung zur EKT bei unter 18-jährigen Patient_innen an drei deutschen universitären Zentren. Hier wurden alle dokumentierten Fälle erfasst und auf Wirksamkeit und Verträglichkeit hin untersucht. Darüber hinaus erfolgte eine umfassende PubMed-basierte Datenbankrecherche. Ergebnisse und Schlussfolgerungen: International existieren keine MetaAnalysen oder kontrollierte randomisierte Studien und aus Deutschland kaum veröffentlichte Fälle zum Thema Elektrokonvulsionstherapie in der Kinder- und Jugendpsychiatrie. Unsere Daten zur EKT bei 29 12- bis 17-jährigen Patient_innen zeigen eine gute Wirksamkeit. Nebenwirkungen traten selten auf. Es ergaben sich keine Hinweise auf Unterschiede bezüglich Indikation (Depression, Katatonie, Schizophrenie), Wirksamkeit, negative Prädiktoren und Verträglichkeit einer EKT zwischen erwachsenen und minderjährigen Patient_innen. Die Ergebnisse deuten zudem darauf hin, dass der Einsatz der EKT bei Adoleszenten früher im therapeutischen Prozess in Erwägung gezogen werden sollte.
\end{abstract}

Schlüsselwörter: Elektrokonvulsionstherapie, EKT, Kinder- und Jugendpsychiatrie, retrospektive Erhebung, minderjährig

Use of electroconvulsive therapy in adolescents - A retrospective survey on 12- to 17-year-old patients at three university hospitals in Germany

Abstract. Background: Electroconvulsive therapy (ECT) is a highly effective and well-researched therapy in adult psychiatry and has been successfully used especially as a treatment for severe depressive, catatonic, and psychotic disorders. Although severe disease progressions also occur in child and adolescent psychiatry, ECT is used much less frequently there. This may be because hardly any data have been collected on the use, effectiveness, and tolerability of ECT in child and adolescent psychiatric patients. This article outlines the application, effectiveness, and tolerability of ECT when applied to young adolescents in Germany. Methods: A retrospective survey on ECT in 29 patients under 18 years of age was conducted at three German university centers. All documented cases were recorded and evaluated for effectiveness and tolerability. In addition, a comprehensive PubMed-based database search was carried out. Results and conclusions: Internationally, there are no meta-analyses or randomized controlled studies and hardly any published cases on electroconvulsive therapy in German child and adolescent psychiatry. Our data on ECT show high efficacy in previously treatment-resistant and severely ill patients. Side effects occurred rarely. There was no evidence of differences between adults and adolescents in indication (depression, catatonia, schizophrenia), effectiveness, tolerability, and negative predictors of response to ECT. The results also suggest that the use of ECT in adolescents should be considered earlier in the treatment course.

Keywords: electroconvulsive therapy, ECT, child and adolescent psychiatry, retrospective survey, adolescents 


\section{Einleitung}

Die Elektrokonvulsionstherapie (EKT) stellt die derzeit wirksamste Behandlungsform schwerer und therapieresistenter Depressionen sowie psychotischer Störungen dar (Fink \& Taylor, 2007; Grözinger, Conca, Nickl-Jockschat \& Di Pauli, 2013; Pagnin, de Queiroz, Pini \& Cassano, 2004). Laut der Nationalen VersorgungsLeitlinie soll die EKT „bei schweren, vital bedrohlichen oder therapieresistenten depressiven Episoden [mit Empfehlungsgrad A] als Behandlungsalternative in Betracht gezogen werden" (Deutsche Gesellschaft für Psychiatrie und Psychotherapie, Psychosomatik und Nervenheilkunde e.V., 2015). Bezogen auf Erkrankungen aus dem schizophrenen Formenkreis sollte "bei eindeutiger medikamentöser Behandlungsresistenz nach adäquater Therapie in ausreichender Dosis und Zeitdauer, [...] eine EKT zur Augmentierung mit dem Ziel der Verbesserung des klinischen Gesamtzustands angeboten werden" (Empfehlungsgrad B; Deutsche Gesellschaft für Psychiatrie und Psychotherapie, Psychosomatik und Nervenheilkunde e.V., 2019).

Obwohl die EKT weltweit zum Einsatz kommt, finden sich in Bezug auf die Häufigkeit der Anwendung starke nationale und zum Teil regionale Unterschiede (Benson-Martin \& Milligan, 2015; Leiknes, Jarosh-von Schweder \& Hoie, 2012; Wang et al., 2015). Während 2008 in Deutschland nur etwas mehr als drei Personen pro 100000 Einwohner mit diesem Verfahren behandelt wurden (Loh, Nickl-Jockschat, Sheldrick \& Grözinger, 2013), waren es z.B. in Schweden 41 (Nordanskog et al., 2015). Der weltweite Durchschnitt dürfte bei ca. 30 liegen (Leiknes et al., 2012).

\section{Datenlage zur EKT bei Kindern, Jugendlichen und Adoleszenten}

Zur Behandlung von Kindern und Jugendlichen unter 18 Jahren mit EKT existieren kaum systematisch erhobene Daten. Eine Literaturrecherche ergab weltweit - ausgenommen von Einzelfallberichten - nur wenige Publikationen, in denen die EKT als Therapieverfahren bei Kindern und Jugendlichen untersucht wurde. Dabei umfassen die meisten Publikationen Fälle von Jugendlichen, die bei EKT-Behandlung mindestens 13 Jahre alt waren (siehe Tabelle 1). Zur EKT bei Kindern existiert neben Einzelfallberichten kaum Literatur. Insgesamt finden sich 594 beschriebene Fälle, davon sind allerdings 411 Fälle einem einzigen medizinischen Zentrum in China zuzuordnen. Ältere amerikanische Arbeiten lassen ebenfalls auf höhere Fallzahlen schließen. So werteten Thompson und Blaine (1987) medizinische Daten zu EKT-Behandlungen in den USA aus und schätzten darauf basierend, dass im Jahre 1975 ca. 3500 Kinder und Jugendliche im Alter zwischen 11 und 20 Jahren mit EKT be- handelt worden seien (Thompson \& Blaine, 1987). W. Vaughn McCall, Herausgeber des Journal of ECT, schätzte 2013 den Anteil Minderjähriger unter allen EKT-Patient_innen in den USA auf ca. 1\% (McCall, 2013). In Schweden wurde im Jahr 2013 an ca. 21 von 100000 adoleszenten Einwohner_innen (15-24 Jahre) eine EKT durchgeführt (Nordanskog et al., 2015). Möglicherweise ist die internationale Erfahrung in diesem Bereich erheblich größer als die wissenschaftlich berichtete Evidenz. Große Unterschiede bei den veröffentlichten Fällen im internationalen Vergleich bezogen auf die Anzahl der Fälle sowie auf die beschriebenen Parameter erschweren eine Vergleichbarkeit der gefundenen Daten. Eine Schwierigkeit in der Literaturrecherche stellt hier z.B. der Umstand dar, dass sich viele Veröffentlichungen zur EKT bei Adoleszenten auf Daten von Patient innen beziehen, die bei EKT-Beginn unter 21 oder sogar unter 25 Jahre alt waren. Dadurch ist es nur eingeschränkt möglich, aus diesen Daten den Anteil der unter 18-Jährigen zu bestimmen. Einen Überblick über die in der Literatur gefundenen Fallserien gibt Tabelle 1.

Betrachtet man die Datenlage in Deutschland, findet man derzeit nur zwei Einzelfallberichte sowie eine Fallserie $(n=4)$ aus der gleichen Arbeitsgruppe, die sich mit EKT bei katatonen, schizophrenen und psychotisch depressiven Minderjährigen befassen (Häßler et al., 2013; Reinhardt, Wunsch, Weirich, Häßler \& Buchmann, 2018; Weirich et al., 2020).

Über Erhaltungstherapie mit EKT (E-EKT; einzelne EKT-Behandlungen, die im Anschluss an eine Serienbehandlung zur Aufrechterhaltung des Therapieerfolgs mit zeitlichen Abständen von in der Regel 1 bis 4 Wochen durchgeführt werden) bei Kindern und Jugendlichen wird in der Literatur noch spärlicher berichtet. Es findet sich nur eine Fallserie mit sechs Jugendlichen aus den USA, die nach einer schweren depressiven Episode mit E-EKT behandelt wurden, in fünf von sechs Fällen mit gutem Erfolg (Ghaziuddin, Dumas \& Hodges, 2011).

Insgesamt ist von einem deutlich geringeren Einsatz der EKT bei Kindern und Jugendlichen im Vergleich zu Erwachsenen auszugehen, was einerseits mit dem zurückhaltenden Einsatz dieser Methode bei dieser Patient_innengruppe, andererseits mit der geringen Prävalenz von z.B. schweren Schizophrenien, Katatonien oder affektiven Erkrankungen bei Kinder und Jugendlichen zu erklären sein könnte.

\section{Empfehlung der Anwendung von EKT bei Kindern, Jugendlichen und Adoleszenten}

In den schon etwas älteren Behandlungsrichtlinien der American Academy of Child and Adolescent Psychiatry zum Einsatz der Elektrokonvulsionstherapie bei Jugendli- 
Tabelle 1. Übersicht der Publikationen zur Anwendung der Elektrokonvulsionstherapie (EKT) bei Kindern, Jugendlichen und Adoleszenten (ausgenommen: Einzelfallberichte).

\begin{tabular}{|c|c|c|c|c|}
\hline Publikation & $\begin{array}{l}\text { Patienten } \\
(n)\end{array}$ & Land & Zeitraum & $\begin{array}{l}\text { Altersspanne } \\
\text { und Besonderheiten }\end{array}$ \\
\hline Baeza et al., 2010 & 13 & Spanien & $2003-2007$ & $\begin{array}{l}13-17 \text { Jahre } \\
1 \text { prolongierter Anfall }\end{array}$ \\
\hline Benson et al., 2019 & 16 & USA & $2011-2016$ & 16-25 Jahre \\
\hline Bloch, Sobol, Levko-vitz, Kron \& Ratzoni, 2008 & 13 & Israel & 5 Jahre & 13-20 Jahre \\
\hline Duffett, Hill \& Lelliott, 1999 & 12 & England & 1996 & Bis 17 Jahre \\
\hline Grover et al., 2013 & 39 & Indien & $1999-2011$ & 13-18 Jahre \\
\hline Jacob et al., 2014 & 22 & Indien & 10 Jahre & Bis 16 Jahre \\
\hline Karayağmurlu et al., 2020 & 78 & Türkei & $2011-2017$ & $\begin{array}{l}\text { Mittleres Alter } 17.1+/-1 \\
6 \text { prolongierte Anfälle } \\
4 \times \text { Switch in eine Manie }\end{array}$ \\
\hline Loiseau, Harrisson, Beaudry \& Patry, 2017 & 6 & Kanada & $1995-2014$ & 15-17 Jahre \\
\hline Mitchell, Hassan \& Ghaziuddin, 2018 & 25 & USA & $1989-2015$ & $\begin{array}{l}\text { Jünger als } 18 \text { Jahre. Follow-up im Alter von } \\
21.1 \text { Jahren, } 44 \text { Monate nach letzter EKT } \\
\text { (Durchschnitt) }\end{array}$ \\
\hline Nordanskog et al., 2015 & *21/100000 & Schweden & 2013 & 15-24 Jahre \\
\hline Paillère-Martinot, Zivi \& Basquin, 1990 & 9 & Frankreich & 3 Jahre & 15-19 Jahre \\
\hline Puffer et al., 2016 & 51 & USA & $1991-2013$ & Mittleres Alter 16.8, +/-1.6 \\
\hline Rootes-Murdy et al., 2019 & 9 & USA & $2007-2014$ & Bis 17 Jahre \\
\hline Stein et al., 2004 & 36 & Israel & $1991-1997$ & 13-19 Jahre \\
\hline Wang, Yang, Jia, Zhou \& Zheng, 2018 & 411 & China & $2007-2016$ & $\begin{array}{l}\text { 13-17 Jahre } \\
\text { Fraglich hohe Anzahl }\end{array}$ \\
\hline Zhand, Courtney \& Flament, 2015 & 13 & Kanada & $2008-2013$ & 15-18 Jahre \\
\hline
\end{tabular}

Anmerkung: *21/100 000 Einwohner_innen im Alter von 15 bis 24 Jahre erhielten im Jahr 2013 eine EKT.

chen von 2004 (Ghaziuddin, Kutcher, Knapp \& Bernet, 2004; Ghaziuddin, Kutcher, Knapp \& the Work Group on Quality Issues, 2004) wird empfohlen, in einer „entsprechenden klinischen Situation den Einsatz [einer EKT] zu erwägen“. Demzufolge sind die drei folgenden Indikationskriterien zu prüfen (Benson \& Seiner, 2019):

- Diagnose: anhaltend schwere depressive Störung oder Manie mit oder ohne psychotische Symptome, schizoaffektive Störungen oder Schizophrenien. Empfohlen wird ebenfalls der Einsatz zur Behandlung einer Katatonie und des malignen neuroleptischen Syndroms.

- Schweregrad der Symptomatik: Es muss eine anhaltend schwere und beeinträchtigende Symptomatik vorliegen. Diese kann lebensbedrohlich sein, wie z.B. die Verweigerung von Essen oder Trinken, aber auch akute Suizidalität, unkontrollierbare Manie oder floride psychotische Symptomatik.
- Fehlendes Ansprechen auf bisherige Behandlungsversuche/Therapieresistenz: Nichtansprechen auf zwei adäquate Psychopharmaka begleitet von anderen angemessenen Behandlungsmodalitäten.

Ein früherer Einsatz der EKT sollte bedacht werden, wenn

- adäquate Medikationsversuche nicht möglich sind aufgrund von Unverträglichkeitsproblemen.

- die Patientin/der Patient schwer beeinträchtigt ist und es somit nicht möglich ist, Medikamente zu verabreichen.

- ein Abwarten des Ansprechens auf Psychopharmaka potenziell lebensbedrohlich ist.

Ähnlich dazu empfiehlt die deutsche, derzeit sich in Überarbeitung befindende S3-Leitlinie zur Behandlung von Kindern und Jugendlichen mit depressiven Störungen, dass bei Jugendlichen „eine Anwendung [der EKT] bei 
Fällen mit sehr schweren Formen der Depression, bei denen die in dieser Leitlinie empfohlenen Ansätze keine Wirkung gezeigt haben, in Erwägung gezogen werden kann" (Deutsche Gesellschaft für Kinder- und Jugendpsychiatrie, Psychosomatik und Psychotherapie e.V., 2013). Eine EKT bei Kindern wird aufgrund des Fehlens , aussagekräftiger Untersuchungen" zu dem Thema in der S3Leitlinie nicht empfohlen; die Datenlage zu EKT bei unter 13-Jährigen ist, wie bereits oben beschrieben, sehr dünn.

Hinsichtlich der Durchführung der EKT bei Kindern und Jugendlichen existiert keine Evidenz für ein unterschiedliches Vorgehen im Vergleich zur Durchführung bei erwachsenen Patient_innen z.B. in Bezug auf klinische Voruntersuchungen, Behandlungsfrequenz, Behandlungsanzahl, anästhesiologische Medikation, Nachbehandlung mit Psychopharmaka oder Erhaltungs-EKT.

Dennoch sind wenige Besonderheiten für die EKT von Kindern und Jugendlichen hervorzuheben:

- Die Indikation stellt eine Fachärztin/ein Facharzt für Kinder- und Jugendpsychiatrie.

- Sowohl Patient_innen als auch deren Sorgeberechtige werden über die Behandlung aufgeklärt, eine Zustimmung erfolgt ebenfalls durch die Patient_innen und die Sorgeberechtigen.

- Da jüngere Patient_innen eine niedrigere Krampfschwelle haben, sollte die initiale Stimulusintensität möglichst gering gehalten werden (in der Regel Start mit $5 \%$ ). Aus diesem Grund muss aber auch besonders auf das Auftreten prolongierter Anfälle geachtet werden.

Bezogen auf die Wirksamkeit finden sich bei Kindern und Jugendlichen ähnlich hohe Raten des Ansprechens auf eine EKT wie bei Erwachsenen. In der Literatur werden Responseraten von 50 bis $100 \%$ beschrieben, wobei Patient_innen mit einer affektiven Erkrankung mit einer höheren Wahrscheinlichkeit auf die Behandlung respondieren als Patient_innen mit einer Erkrankung aus dem schizophrenen Formenkreis (Ghaziuddin, Kutcher, Knapp \& the Work Group on Quality Issues, 2004; Grover et al., 2013; Walter, Koster \& Rey, 1999). Wie auch bei erwachsenen Patient_innen scheint selbstverletzendes Verhalten bei Kindern und Jugendlichen ein negativer Prädiktor für die Wirksamkeit der EKT zu sein (Rootes-Murdy et al., 2019).

Die Häufigkeit und die Intensität von Nebenwirkungen unter der EKT scheinen bei Kindern und Jugendlichen vergleichbar mit dem Nebenwirkungsprofil bei Erwachsenen zu sein (Shoirah \& Hamoda, 2011). Ein vermehrtes Auftreten von prolongierten und tardiven Anfällen wird diskutiert (Shoirah \& Hamoda, 2011) und ist aufgrund der niedrigeren Krampfschwelle auch plausibel, wobei beide Nebenwirkungen aber auch bei Erwachsenen auftreten (Ghaziuddin, Kutcher, Knapp \& the Work Group on Quality Issues, 2004). Generell scheinen jüngere Patient_innen auch häufiger unter gut behandelbaren und nur kurz anhaltenden postiktalen Kopfschmerzen zu leiden. Da eine große Datenbasis zu Nebenwirkungen unter EKT bei Kindern und Jugendlichen fehlt, kann hier keine abschließende Aussage zur Häufigkeit getroffen werden.

Der insgesamt begrenzten Datenlage zur EKT im Kinder- und Jugendbereich steht ein Interesse seitens der Behandler_innen entgegen. So kam eine groß ausgelegte Umfrage unter Kinder- und Jugendpsychiatern in Belgien zu dem Ergebnis, dass sich nur 1\% der Befragten gut mit einer EKT-Behandlung von Minderjährigen auszukennen glaubt, 91\% aber gern ein größeres Wissen bezüglich dieses Themas hätten (De Meulenaere, De Meulenaere, Ghaziuddin \& Sienaert, 2018).

Ziel der vorliegenden Arbeit ist die retrospektive Darstellung und Analyse der bislang größten Fallsammlung zur Anwendung der EKT bei Kindern und Jugendlichen im deutschsprachigen Raum. Dafür wurden alle Daten zu durchgeführten EKTs bei Kindern und Jugendlichen an den universitären Zentren Rostock, Mannheim und Göttingen aus den letzten 10 Jahren erfasst.

\section{Material und Methoden}

Es wurden retrospektiv Patient_innen an den drei universitären kinder- und jugendpsychiatrischen Zentren erfasst, die bis einschließlich 2019 eine EKT-Serienbehandlung erhielten und zu Beginn der Behandlung unter 18 Jahre alt waren. Insgesamt konnten 29 Patient_innen in die Erhebung eingeschlossen werden, von denen vier Patient_innen jeweils zwei EKT-Serien erhielten. Eine erneute EKTSerie ist hier definiert durch einen klinischen Rückfall. Dieser kann zu jedem Zeitpunkt nach einer ersten EKTSerie auftreten.

Anhand der Patient_innenakten wurden erfasst: die zur EKT führende Diagnose, Nebendiagnosen, Alter der Patient_innen bei Behandlungsbeginn, Geschlecht, Erkrankungsschwere vor und nach EKT-Serie (Clinical Global Impression Severity Scale [CGI-S]; Guy, 1976), IQ, Anzahl der erfolglosen medikamentösen Behandlungen der letzten 6 Monate vor EKT-Serie sowie die aktuelle Medikation vor und nach der EKT-Serie. Bezogen auf die EKT-Durchführung wurden erfasst: Elektrodenposition, Ladungsmenge, Narkosemittel, Anzahl der EKT-Behandlungen, aufgetretene Nebenwirkungen unter EKT und ob im Anschluss eine Erhaltungs-EKT erfolgte.

Die Statistik erfolgte zunächst rein deskriptiv (mit Angabe der Mittelwerte und Standardabweichungen) sowie in Bezug auf ein Ansprechen auf die Behandlung (definiert als ein finaler CGI-S kleiner oder gleich 3) als logistische Regressionsanalyse mit Geschlecht, Elektrodenposition, 
Ladungsmenge, Anzahl der erfolglosen medikamentösen Therapieversuche der letzten 6 Monate und Anzahl der im Rahmen der EKT-Serie durchgeführten Einzelbehandlungen als Kovariate (STATA Version 15.1, Signifikanzlevel 0.05).

\section{Ergebnisse}

Die deskriptiven Ergebnisse der 29 Patient_innen sind in Tabelle 2 dargestellt. Von den vier Patient_innen, die zweimal eine EKT-Serie erhielten (im Durchschnitt 1 Jahr später), wurde in der Tabelle und in der Statistik jeweils nur die erste EKT-Serienbehandlung erfasst.

Keine der in Tabelle 2 genannten Variablen zeigt einen signifikanten Mittelwertsunterschied zwischen den drei häufigsten Hauptdiagnosen (Depression [ $n=13]$, Katatonie $[n=6]$ und Schizophrenie $[n=8])$. Zwei Patient innen wurden aufgrund organisch bedingter Depression und Verhaltensauffälligkeiten behandelt.

\section{Wirkung}

Die Veränderung der Schwere der Erkrankung der Patient_innen wurde mittels CGI-S vor und nach der EKT-Serie ermittelt. Auf der siebenstufigen Skala des CGI-S lässt sich die Symptomschwere einer Patientin/eines Patienten beschreiben; eine 1 entspricht dem Zustand „nicht erkrankt" und eine 7 ,gehört zu den am schwersten erkrankten Patient_innen". Insgesamt kam es unter der EKT im Durchschnitt nach 4.6 erfolglosen medikamentösen Therapieversuchen in den letzten 6 Monaten zu einer hoch signifikanten Verbesserung $(t=10.3, p<.00001)$ um 3.2 Punkte im CGI-S (siehe auch Abbildung 1). Keine/keiner der behandelten Patient_innen zeigte eine Verschlechterung der Symptomatik.

Hinsichtlich des Therapieansprechens zeigte sich in der logistischen Regression eine signifikante Abhängigkeit von der Anzahl der erfolglosen medikamentösen Therapien in den letzten 6 Monaten $(p=.027$; siehe auch Abbildung 2). Für die anderen in Tabelle 2 genannten Parameter zeigten sich hingegen keine signifikanten Korrelationen mit der klinischen Verbesserung.

Fünf der Patient_innen hatten als Nebendiagnose die Diagnose einer Anorexie, davon unabhängig drei weitere Patient innen die Nebendiagnose einer emotional instabilen Persönlichkeitsstörung. Von diesen acht Patient innen mit der Nebendiagnose Persönlichkeitsstörung/Anorexie respondierten nur einer auf die Behandlung. Trotzdem betrug die Verbesserung im CGI-S im Durchschnitt dieser acht Patient_innen noch 2.75. Im exakten Test nach Fischer zeigte sich ein signifikanter Unterschied $(p=.044)$ in der Response zwischen diesen acht vs. den restlichen 21 Patient_innen.

Tabelle 2. Deskriptive Ergebnisse der 29 mit Elektrokonvulsionstherapie (EKT) behandelten Patient_innen.

\begin{tabular}{|c|c|c|c|c|c|}
\hline & Mittelwert & $S D$ & $n$ & Min & $\operatorname{Max}$ \\
\hline Alter $(J)$ & 15.4 & 1.3 & 29 & 12 & 17 \\
\hline Geschlecht $(m=1, w=0)$ & 0.31 & 0.47 & 29 & 0 & 1 \\
\hline CGI-S initial & 6.89 & 0.31 & 29 & 6 & 7 \\
\hline CGI-S final & 3.69 & 1.65 & 29 & 1 & 7 \\
\hline CGI-S Differenz & 3.21 & 1.68 & 29 & 0 & 6 \\
\hline $1 Q$ * & 96.2 & 20.8 & 26 & 30 & 139 \\
\hline Ladungsmenge (\%) & 48 & 37 & 29 & 5 & 140 \\
\hline Anzahl der EKT-Behandlungen & 18.2 & 7.8 & 28 & 7 & 37 \\
\hline Anschließende Erhaltungs-EKT & 0.14 & 0.35 & 29 & 0 & 1 \\
\hline Response (CGI-S final <4) & 0.45 & 0.51 & 29 & 0 & 1 \\
\hline Anzahl abgesetzter Medikamente & 0.79 & 1.32 & 29 & -1 & 4 \\
\hline Anzahl medikamentöser Therapien in den letzten 6 Monaten & 4.6 & 3.1 & 29 & 0 & 15 \\
\hline
\end{tabular}

Anmerkungen: CGI-S = Clinical Global Impression Severity Scale.

* IQ: Neun Patient_innen wurden nicht getestet und bei klinisch normaler Intelligenz mit 100 einberechnet. Eine Patient/ein Patient mit klinisch deutlich überdurchschnittlichem IQ wurde mit 120 geschätzt. Zwei ebenfalls nicht getestete Patient_innen mit klinisch leichter und schwerer Intelligenzminderung wurden mit jeweils 60 und 30 Punkten einberechnet. 


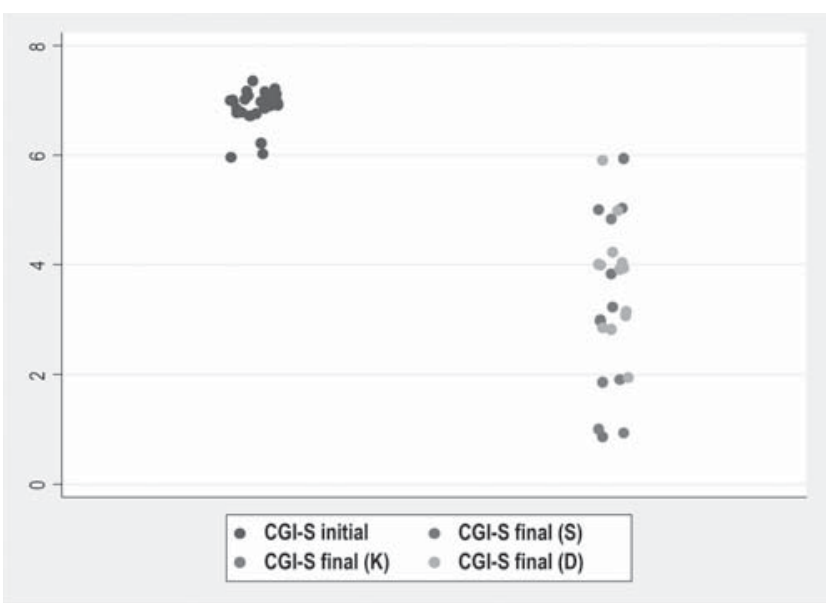

Abbildung 1. Erkrankungsschwere vor und nach Elektrokonvulsionstherapie (EKT). Der erreichte CGI-S-Wert (Clinical Global Impression Severity Scale) für Patient_innen mit der Hauptdiagnose Schizophrenie (S), Katatonie (K) und Depression (D).

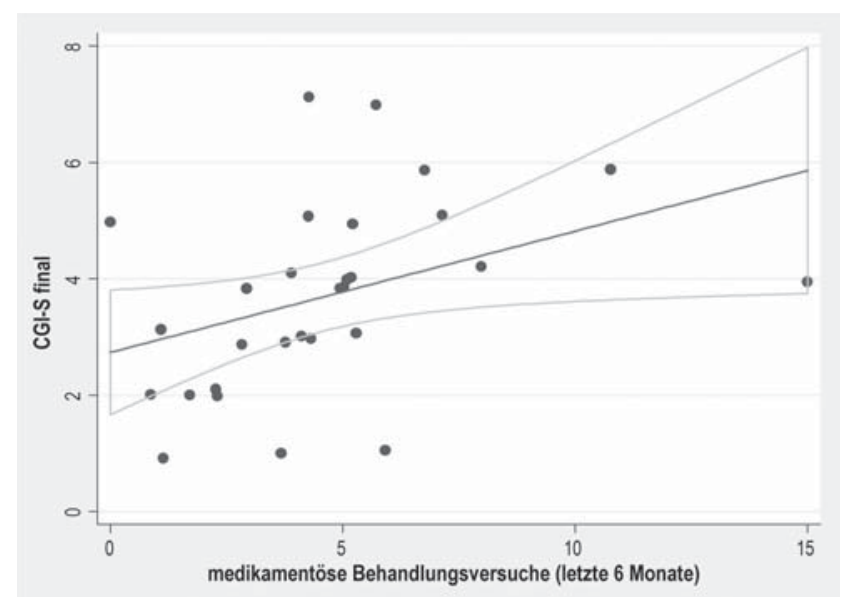

Abbildung 2. Erkrankungsschwere nach Elektrokonvulsionstherapie (EKT) in Abhängigkeit von der Anzahl der erfolglosen medikamentösen Therapien in den letzten 6 Monaten. Der erreichte CGI-S-Wert (Clinical Global Impression Severity Scale) korreliert (nach Spearman) mit der Anzahl der erfolglosen medikamentösen Therapien in den letzten 6 Monaten mit einem $p=.007$.

An weiteren Nebendiagnosen wurde bei zwei Patient innen zusätzlich eine hyperkinetische Störung und bei zwei weiteren ein Autismus diagnostiziert.

\section{Nebenwirkungen}

Fünf der Patient_innen beschrieben kognitive Nebenwirkungen in Form von Kurzzeitgedächtnisstörungen unter der Behandlung, welche auch durch die klinische Beobachtung bestätigt wurden. Diese sistierten laut Patient_innenwahrnehmung als auch im klinischen Ein- druck nach Beendigung der EKT bei vier der Patient innen. Nur in einem Fall blieben die unter der Serie aufgetretenen Kurzzeitgedächtnisstörungen auch während der anschließenden Erhaltungs-EKT in reduzierter Form bestehen (wobei die Erkrankung ebenfalls keine Vollremission erreichte). Die dokumentierten kognitiven Defizite wurden in allen fünf Fällen rein deskriptiv erfasst, neuropsychologische Untersuchungen wurden nicht vorgenommen.

Bei zwei Patient_innen kam es jeweils einmalig zu einem prolongierten Anfall, zwei Patient_innen berichteten von kurzzeitigen Kopfschmerzen und eine Patientin/ein Patient zeigte eine leichte allergische Reaktion auf die Anästhetika. Bei 19 Patient_innen (66\%) wurden keinerlei Nebenwirkungen dokumentiert.

\section{Elektrodenposition}

Betrachtet man nur die finale Elektrodenposition (bei drei Patient innen kam es zu einem Wechsel der Elektrodenposition während der EKT-Serie), so wurden 17 Patient_innen rechts unilateral, zehn Patient_innen bitemporal (= bilateral) und zwei Patient_innen links anterior, rechts temporal (LART) behandelt.

\section{Narkose}

Barbiturate: Drei Patient_innen wurde als Narkosemittel Thiopental, einer Patientin/einem Patienten Methohexital verabreicht. Zwei weitere erhielten initial Methohexital und später Propofol (Methohexital ist im Handel seit 2019 nicht mehr verfügbar). Bei 13 Patient_innen erfolgte die Narkose mit S-Ketamin, bei fünf mit Ketofol (einer Mischung aus S-Ketamin und Propofol) und bei sieben mit Etomidate.

\section{Begleitmedikation unter der EKT-Serie}

Von den 13 depressiven Patient_innen erhielten initial elf Antidepressiva, fünf Antipsychotika (bis zu drei verschiedene Präparate), zwei Phasenprophylaktika und sieben Benzodiazepine. Von den insgesamt neun schizophrenen Patient_innen erhielten alle Antipsychotika (bis zu vier verschiedene Präparate) und fünf Benzodiazepine. Von den katatonen Patient_innen erhielten alle sechs Benzodiazepine, drei Neuroleptika und eine/einer ein Phasenprophylaktikum.

Nach Abschluss der EKT-Serie erhielt keine Patientin/ kein Patient mehr als zwei verschiedene Antipsychotika. Nur zwei Patient_innen erhielten noch ein Benzodiazepin. 


\section{Diskussion}

In unserer retrospektiven Analyse erwies sich die EKT bei 29 medikamentös therapieresistenten minderjährigen Patient_innen in Deutschland als diagnoseübergreifend effektives und nebenwirkungsarmes therapeutisches Verfahren. Obwohl die EKT in der Adoleszenz international und explizit in vielen westlichen und asiatischen Ländern ein anerkanntes Verfahren ist, existieren kaum Daten zur Anwendung in Deutschland. Somit kann eine klinische Unterversorgung junger, psychiatrisch schwerstkranker Patient_ innen oder zumindest ein Mangel an vorgestellten Fällen in Deutschland angenommen werden; dementsprechend leisten die hier vorgestellten 29 Therapieverläufe einen wichtigen Beitrag zur publizierten Gesamtevidenz (siehe Tabelle 1).

\section{Wirksamkeit}

In der Erwachsenenpsychiatrie zeigt die EKT eine hohe Wirksamkeit zur Behandlung depressiver und psychotischer Erkrankungen und wird somit gerade bei schweren Krankheitsverläufen empfohlen und häufig angewendet (Bahji, Hawken, Sepehry, Cabrera \& Vazquez, 2019; Kho, van Vreeswijk, Simpson \& Zwinderman, 2003; Lally et al., 2016; Wang, Zheng et al., 2018).

Unsere Ergebnisse zeigen eine vergleichbar hohe Wirksamkeit der EKT als Anwendung in der Kinder- und Jugendpsychiatrie. Bei den von uns untersuchten Behandlungsverläufen verbesserte sich der CGIS im Mittel hoch signifikant um 3.2 Punkte und $45 \%$ der Patient_innen erreichten einen CGI-S kleiner oder gleich $\overline{3}$ („leicht erkrankt"), obwohl sich alle Patient_ innen bereits länger als 6 Monate (teilweise seit Jahren) in der gleichen Erkrankungsperiode mit schwerer Symptomatik (CGI-S = 6.9 Punkte, 7 bedeutet: ,gehört zu den am schwersten erkrankten Patient_innen") befanden und alleine in diesen 6 Monaten im Durchschnitt 4.6 „erfolglose“ medikamentöse Therapien erhalten hatten.

In der Erwachsenenpsychiatrie erwiesen sich Persönlichkeitsstörungen als negativer Prädiktor der Therapieresponse (Feske et al., 2004; Popiolek et al., 2019). Unsere Daten weisen in eine ähnliche Richtung: Von den Patient innen mit der Nebendiagnose Persönlichkeitsstörung oder Anorexie sprachen nur $12.5 \%$ (eine/einer von acht Patient innen) gut auf die Behandlung an, wohingegen die Response bei den Patient_innen ohne diese Nebendiagnose signifikant höher bei $57 \%$ lag.

Als signifikanter Einflussfaktor auf die Wirksamkeit der EKT zeigte sich in unserer Studie auch die Anzahl der vorangegangenen Therapieversuche; hier fanden wir eine Korrelation zwischen schlechterer Therapieresponse und einer vergleichbar hohen Anzahl von Therapieversuchen in den letzten 6 Monaten vor EKT (Abbildung 2). Hieraus lässt sich ableiten, dass eine EKT früher zum Einsatz kommen sollte, denn in Übereinstimmung mit der Erfahrung im Erwachsenenbereich neigen die Erkrankungen zur Chronifizierung und damit allgemein zu einer schlechteren Therapieresponse, auch bezogen auf die EKT (Loo, Mahon, Katalinic, Lyndon \& Hadzi-Pavlovic, 2011) .

\section{Bewertung der EKT durch Patient_innen}

Eine Befragung unter mit EKT behandelten adoleszenten Patient_innen ergab eine positive Bewertung der EKT; sie wurde als eine effektive Therapieform eingeschätzt. Auch zeigte sich, dass eine ablehnende Haltung der Behandlungsmethode gegenüber seltener auftrat als bei einer Vergleichsgruppe adoleszenter Patient_innen ohne EKT-Erfahrung ( $8 \%$ vs. $28 \%$; Bloch et al., 2018). Die Fallzahl war mit 13 EKT-Patient_innen allerdings gering, sodass es schwierig ist, das Ergebnis der Befragung z.B. als Grundlage für eine Therapieentscheidung $\mathrm{zu}$ berücksichtigen.

\section{Nebenwirkungen}

Bei erwachsenen Patient_innen zeigen sich bei ca. einem Drittel klinisch relevante, reversible kognitive Nebenwirkungen durch die EKT (Sackeim, Ross, Hopkins, Calev \& Devanand, 1987). Sie werden zum einen durch die Patient_innen selbst wahrgenommen und können z.B. durch strukturierte Interviews erfasst werden (Sackeim et al., 1987). Zum anderen lassen sich kognitive Defizite in verschiedenen kognitiven Domänen messen und sind in der Regel nach 2 bis 3 Wochen nicht mehr nachweisbar; für einen umfassenden Überblick lässt sich hier eine Metaanalyse von Semkovska und McLoughlin (2010) heranziehen, die 84 Studien $(n=2981)$ zu kognitiven Defiziten unter EKT, gemessen mittels 22 verschiedener standardisierter neuropsychologischer Testverfahren, auswertet und vergleicht. Die Inzidenz reversibler kognitiver Nebenwirkungen ist zudem möglicherweise altersabhängig, sicherlich aber zunehmend bei älteren Patient_innen mit komorbiden neurodegenerativen Erkrankungen (z. B. gezeigt anhand der Ergebnisse des Mini Mental Status Tests vor und nach EKT bei nicht/mild kognitiv vorgeschädigten im Vergleich zu dementen Patient_innen; Hausner, Damian, Sartorius \& Frölich, 2011). Demnach erscheint es stimmig, dass in unserer Untersuchung deutlich weniger als ein Drittel der adoleszenten Patient_innen, näm- 
lich nur 5 von 29, unter reversiblen kognitiven Nebenwirkungen litten.

Vergleichbar dazu ließen sich in einer spanischen Follow-up-Studie 2 Jahre nach einer EKT-Serie bei neun adoleszenten schizophrenen Patient_innen im Vergleich zu einer medikamentös behandelten Gruppe weder klinisch noch neuropsychologisch (standardisierte Testung von Arbeits- und verbalem Gedächtnis, Aufmerksamkeit und Exekutivfunktionen) kognitive Unterschiede feststellen (de la Serna et al., 2011).

Die Inzidenz postiktaler Kopfschmerzen ist sehr heterogen und wird im Mittel von ca. 30\% aller erwachsenen Patient_innen angegeben (Mulder \& Grootens, 2020). Einzelne kleinere Studien zeigen deutlich höhere Inzidenzen bei Jugendlichen (Ghaziuddin et al., 1996). In unserer Untersuchung zeigte sich diese Nebenwirkung mit zwei von 29 vergleichsweise selten. Bezüglich postiktaler Kopfschmerzen ist es für Behandler_innen sowie Patient_innen wissenswert, dass sie benigner Art und mittels nichtsteroidaler Entzündungshemmer gut zu behandeln sind (Sartorius, Pycha, Grözinger \& Conca, 2013).

Die Inzidenz prolongierter (und tardiver) epileptischer Anfälle scheint im jüngeren Alter mit 8 bis $63 \%$ (Karayağmurlu et al., 2020; Puffer, Wall, Huxsahl \& Frye, 2016) häufiger zu sein als bei Erwachsenen mit ca. $1 \%$ (häufiger bei der ersten EKT; Glénisson et al., 1998). Auch in unserer Untersuchung zeigten zwei von 29 Patient_innen (7\%) einmalig einen prolongierten epileptischen Anfall. Das ist in Übereinstimmung mit einer niedrigeren Krampfschwelle bei jüngeren Patient_innen. Dabei ist darauf zu achten, dass bei einer niedrigeren Krampfschwelle auch mit einer niedrigeren „Stimulationsenergie“ (exakt: Ladungsmenge) behandelt werden kann, zum anderen steigt aber bei einer zu niedrigen Ladungsmenge das Risiko prolongierter oder tardiver Anfälle. Dazu passt, dass bei einem der beiden berichteten Fälle mit einer Ladungsmenge von nur $10 \%(=50.4 \mathrm{mC})$ stimuliert wurde. So gesehen sollte möglicherweise auf eine deutlich überschwellige Stimulation (z.B. sechsmal über der Krampfschwelle bei rechts unilateraler Stimulation) bei Patient_innen im Kindes- und Jugendalter besonders geachtet werden.

Dass bei zwei Drittel der Patient_innen keine Nebenwirkungen auftraten und es sich bei allen anderen Nebenwirkungen um reversible Phänomene handelte, sollte bei zudem hoher Effektivität und unter Berücksichtigung einer Nutzen-Risiko-Abwägung dazu führen, in ähnlich gelagerten Fällen die EKT-Indikation früher zu stellen und einer weiteren medikamentösen Therapie vorzuziehen. Dennoch ist abschließend auf die Notwendigkeit hinzuweisen, die Patient_innen und ihre Angehörigen im Vorfeld der Behandlung umfassend über den transienten Charakter der Nebenwirkungen aufzuklären, um Ängsten vorzubeugen (Sartorius et al., 2013).

\section{Narkose}

Anästhesist_innen setzen bei Kindern und Jugendlichen deutlich häufiger S-Ketamin als Kurzzeitnarkotikum ein als bei Erwachsenen. Auch im Erwachsenenbereich kommt es derzeit zu einem zunehmenden Einsatz von SKetamin bei EKT-Narkosen (Janke et al., 2015), da es gut vertragen wird (Sartorius, Aksay, Bumb, Janke \& Kranaster, 2015) und sich positiv auf die Krampfqualität auswirkt (Hoyer, Kranaster, Janke \& Sartorius, 2014). Barbiturate (also Thiopental und Methohexital) kommen kaum noch zum Einsatz, da Methohexital derzeit nicht mehr im Handel verfügbar ist und Thiopental aufgrund einer Herstellungsproblematik nur noch eine eingeschränkte Zulassung besitzt. Etomidate wird aufgrund seiner supprimierenden Wirkung auf die Hypothalamus-Hypophysen-Nebennierenrinden-Achse ebenfalls seltener für Kurzzeitnarkosen eingesetzt (Dumps, Colkenius \& Halbeck, 2017). Propofol ist zwar ein generell gut geeignetes Medikament für Kurzzeitnarkosen, hat aber von allen hier genannten Präparaten die stärkste antikonvulsive Potenz, was den Einsatz bei einer EKT erschwert. Ein zukünftiger Kompromiss in diesem Bereich könnte der vermehrte Einsatz von Mischnarkosen bei der EKT sein, z.B. durch die Verwendung von Ketofol (eine Mischung aus Propofol und S-Ketamin). Abschließend ist festzustellen, dass die Verteilung der Narkosemittel in der Kinder- und Jugendpsychiatrie der in der Erwachsenenpsychiatrie entspricht. Die Verteilung der häufig eingesetzten Präparate wird in unserer Stichprobe plausibel abgebildet.

\section{Elektrodenposition}

Es wurden bei 27 von 29 Patient_innen die auch international (im Erwachsenenbereich) am häufigsten verwendeten Elektrodenpositionen verwendet, nämlich rechts unilateral oder bitemporal (= bilateral). Bei entsprechend gewählter Ladungsmenge ist die Effektivität der beiden Verfahren gleichwertig (Semkovska et al., 2016). Die mittlere verwendete Ladungsmenge unterschied sich in unserer Untersuchung nicht zwischen diesen beiden Elektrodenpositionen. Bei rechts unilateraler Stimulation ist zwar die Krampfschwelle niedriger, es sollte jedoch allgemein höher (> 2.56x) über der Krampfschwelle stimuliert werden als bei bitemporaler Stimulation (@ 1.5x), was den insgesamt fehlende Unterschied der mittleren Ladungsmenge erklären könnte. Dass insgesamt 10 von 29 Patient_innen bitemporal behandelt wurden, dürfte durch die überdurchschnittliche Schwere der Erkrankung des behandelten Patient_innenkollektivs zu erklären sein. Hier wäre es zu erwarten, dass die bitemporal behandelten Patient_innen in unserer Studie vor EKT signifikant schwerer krank waren, als die 
rechts unilateral behandelten. Dies bildete sich jedoch nicht im CGI-S ab. Die Tatsache könnte zum einen dem Deckeneffekt, zum anderen womöglich auch der fehlenden Trennschärfe des Messinstruments geschuldet sein.

\section{Medikation vor und nach der EKT-Serie}

Bei den von uns untersuchten Fällen bestand die Medikation vor der EKT-Serie in den meisten Fällen aus mehreren Substanzgruppen. Es ist allgemein bekannt und belegt, dass der Großteil der Patient_innen, die mit einer EKT behandelt werden, einer erheblichen Komedikation verschiedenster Psychopharmaka unterliegen (Bundy, Hewer, Andres, Gass \& Sartorius, 2010), was womöglich die Schwere der Erkrankungen ein Stück weit widerspiegelt.

In unserer Studie konnte durch die EKT eine deutliche Reduktion von Benzodiazepinen (bezogen auf Häufigkeit und Dosierung) erreicht werden, in Einzelfällen konnte auch die vorbestehende Polypharmazie reduziert werden. In vielen Fällen wurde die Medikation angepasst oder auch durch verträglichere Substanzen ersetzt. Dass die Medikation im Durchschnitt (über alle Patient_innen) nur um ein Medikament reduziert werden konnte, liegt wahrscheinlich größtenteils an der initialen Schwere der Erkrankungen, die leitlinienkonform einen entsprechenden Rückfallschutz nach EKT auch bei den Respondern erfordert.

\section{Limitationen}

Die vorliegende Arbeit ist zum einen durch ihren retrospektiven Charakter an sich limitiert. Zum anderen gestaltete sich die Datenerhebung sowohl im Vergleich zwischen den drei Zentren als auch innerhalb dieser heterogen und es wurden keine einheitlichen Skalen verwendet. Zudem wurden Patient_innen mit unterschiedlichen Diagnosen mittels EKT behandelt. Abschließend ist die Anzahl der beschriebenen Fälle im internationalen Literaturvergleich zwar hoch, im Vergleich zu beschriebenen Fällen aus dem Erwachsenenbereich aber niedrig. Umso wichtiger erscheint es, in diesem Kontext zukünftig prospektiv und einheitlicher Daten zu erfassen.

\section{Zusammenfassung}

Die retrospektive Analyse von 29 Patient_innen im Kindes- und Jugendalter dreier universitärer Zentren zeigt eine hohe Effektivität und sehr geringe Nebenwirkungen beim Einsatz der EKT bei schwersten psychiatrischen Erkrankungen. Unsere Daten weisen zudem auf die Sinnhaftigkeit eines früheren Einsatzes der EKT bei schweren psychiatrischen Erkrankungen in der Kinder- und Jugendpsychiatrie hin. Frappierend ist der Vergleich mit Schweden, wo bei 21 von 100000 adoleszenten Einwohnern (15-24 Jahre) allein im Jahre 2013 eine EKT durchgeführt wurde. Zusammenfassend lässt sich feststellen, dass es weiterhin keine Evidenz für Unterschiede in Indikation und Durchführung einer EKT bei adulten oder adoleszenten Patient_innen gibt, es umgekehrt jedoch an Studien zur indikationsgeleiteten und optimierten EKT im kinderund jugendpsychiatrischen Bereich mangelt. Dadurch fehlt es den Behandler_innen an Basiswissen über Indikation, Wirksamkeit und Verträglichkeit der EKT als Behandlung bei Kindern und Jugendlichen, was in einer zurückhaltenderen Anwendung der EKT resultieren kann. Auch existieren nur wenige Daten bezüglich einer Bewertung der EKT durch Patient_innen, die mit ihr in Berührung kommen. Zusammenfassend ist ein Forschungsbedarf zu diesem Thema festzustellen, auch um vorhandene Stigmata gegenüber der EKT als Behandlungsmethode bei Minderjährigen - einem besonders vulnerablen Patient_innenkollektiv - abzubauen und die Behandlung den schwer Erkrankten unter ihnen zugänglich zu machen.

\section{Literatur}

Baeza, I., Flamarique, I., Garrido, J. M., Horga, G., Pons, A., Bernardo, M. et al. (2010). Clinical experience using electroconvulsive therapy in adolescents with schizophrenia spectrum disorders. Journal of Child and Adolescent Psychopharmacology, 20, 205-209.

Bahji, A., Hawken, E.R., Sepehry, A.A., Cabrera, C.A. \& Vazquez, G. (2019). ECT beyond unipolar major depression: Systematic review and meta-analysis of electroconvulsive therapy in bipolar depression. Acta Psychiatrica Scandinavica, 139, 214-226.

Benson, N.M. \& Seiner, S.J. (2019). Electroconvulsive therapy in children and adolescents: Clinical indications and special considerations. Harvard Review of Psychiatry, 276, 354-358.

Benson, N. M., Seiner, S.J., Bolton, P., Fitzmaurice, G., Meisner, R.C., Pierce, C. et al. (2019). Acute phase treatment outcomes of electroconvulsive therapy in adolescents and young adults. Journal of ECT, 35, 178-183.

Benson-Martin, J.J \& Milligan, P.D. (2015). A survey of the practice of electroconvulsive therapy in South Africa. Journal of ECT, 31 , 253-257.

Bloch, Y., Linder, M., Kalman, N., Koubi, M., Gal, G., Nitsan, U. et al. (2018). Adult and adolescent patient evaluations of electroconvulsive therapy in comparison to other therapeutic modalities. Journal of ECT, 34, 45-49.

Bloch, Y., Sobol, D., Levkovitz, Y., Kron, S. \& Ratzoni, G. (2008). Reasons for referral for electroconvulsive therapy: A comparison between adolescents and adults. Australasian Psychiatry, 16, 191-194.

Bundy, B. D., Hewer, W., Andres, F.-J., Gass, P. \& Sartorius, A. (2010). Influence of anesthetic drugs and concurrent psychiatric medi- 
cation on seizure adequacy during electroconvulsive therapy. Journal of Clinical Psychiatry, 71, 775-777.

de la Serna, E., Flamarique, I., Castro-Fornieles, J., Pons, A., Puig, O., Andrés-Perpiña, S. et al. (2011). Two-year follow-up of cognitive functions in schizophrenia spectrum disorders of adolescent patients treated with electroconvulsive therapy. Journal of Child and Adolescent Psychopharmacology, 21, 611-619.

De Meulenaere, M., De Meulenaere, J., Ghaziuddin, N. \& Sienaert, P. (2018). Experience, knowledge, and attitudes of child and adolescent psychiatrists in Belgium toward pediatric electroconvulsive therapy. Journal of ECT, 34, 247-252.

Deutsche Gesellschaft für Kinder- und Jugendpsychiatrie, Psychosomatik und Psychotherapie e.V. (Hrsg.). (2013). Behandlung von depressiven Störungen bei Kindern und Jugendlichen. Evidenz- und konsensbasierte Leitlinie (S3). AWMF-Registernummer 028-043. Verfügbar unter: https://www.awmf.org/leitlinien/detail/ll/028-043.html

Deutsche Gesellschaft für Psychiatrie und Psychotherapie, Psychosomatik und Nervenheilkunde e.V. (Hrsg.). (2019). S3-Leitlinie Schizophrenie. Langfassung, Version 1.0. AWMF-Registernummer 038-009. Verfügbar unter: https://www.awmf.org/ leitlinien/detail/ll/038-009.html

Deutsche Gesellschaft für Psychiatrie und Psychotherapie, Psychosomatik und Nervenheilkunde e.V. (Hrsg.). (2015). S3-Leitlinie/Nationale VersorgungsLeitlinie Unipolare Depression. Langfassung, 2. Aufl., Version 5. AWMF-Registernummer nvl005. Verfügbar unter: https://www.awmf.org/leitlinien/detail/ll/ nvl-005.html

Duffett, R., Hill, P. \& Lelliott, P. (1999). Use of electroconvulsive therapy in young people. British Journal of Psychiatry, 175, 228-230.

Dumps, C., Bolkenius, D. \& Halbeck, E. (2017). Medikamente zur intravenösen Narkoseinduktion: Etomidat. Anaesthesist, 66, 969-980.

Feske, U., Mulsant, B.H., Pilkonis, P.A., Soloff, P., Dolata, D., Sackeim, H.A. et al. (2004). Clinical outcome of ECT in patients with major depression and comorbid borderline personality disorder. American Journal of Psychiatry, 161, 2073-2080.

Fink, M. \& Taylor, M.A. (2007). Electroconvulsive therapy: Evidence and challenges. Journal of the American Medical Association, 298, 330-332.

Ghaziuddin, N., Dumas, S. \& Hodges, E. (2011). Use of continuation or maintenance electroconvulsive therapy in adolescents with severe treatment-resistant depression. Journal of ECT, 27, $168-174$.

Ghaziuddin, N., King, C.A., Naylor, M.W., Ghaziuddin, M., Chaudhary, N., Giordani, B. et al. (1996). Electroconvulsive treatment in adolescents with pharmacotherapy-refractory depression. Journal of Child Adolescent Psychopharmacology, 6, 259-271.

Ghaziuddin, N., Kutcher, S.P., Knapp, P. \& Bernet, W. (2004). Summary of the practice parameter for the use of electroconvulsive therapy with adolescents. Journal of the American Academy of Child \& Adolescent Psychiatry, 43, 119-122.

Ghaziuddin, N., Kutcher, S. P., Knapp, P. \& the Work Group on Quality Issues. (2004). Practice parameter for use of electroconvulsive therapy with adolescents. Journal of the American Academy of Child \& Adolescent Psychiatry, 43, 1521-1539.

Glénisson, L., Auriacombe, M., Fernandez, T., Auriacombe, S., Gomez, F., Uzandisaga, D. et al. (1998). [Adverse seizure reactions after electroconvulsive therapy. Study of personal cases and review of the literature]. Encéphale, 24, 1-8.

Grözinger, M., Conca, A., Nickl-Jockschat, T. \& Di Pauli, J. (Hrsg.). (2013). Elektrokonvulsionstherapie kompakt. Berlin: Springer.

Grover, S., Malhotra, S., Varma, S., Chakrabarti, S., Avasthi, A. \& Mattoo, S. K. (2013). Electroconvulsive therapy in adolescents: A retrospective study from north India. Journal of ECT, 29, $122-126$.
Guy, W. (Ed.). (1976). ECDEU assessment manual for psychopharmacology. Publication ADM 76-338. Rockville, MD: US Department of Health, Education, and Welfare.

Häßler, F., Reis, O., Weirich, S., Höppner, J., Pohl, B. \& Buchmann, J. (2013). A case of catatonia in a 14-year-old girl with schizophrenia treated with electroconvulsive therapy. Zeitschrift für Kinder- und Jugendpsychiatrie und Psychotherapie, 41, 69-74.

Hausner, L., Damian, M., Sartorius, A. \& Frölich, L. (2011). Efficacy and cognitive side effects of electroconvulsive therapy (ECT) in depressed elderly inpatients with coexisting mild cognitive impairment or dementia. Journal of Clinical Psychiatry, 72, 91-97.

Hoyer, C., Kranaster, L., Janke, C. \& Sartorius, A. (2014). Impact of the anesthetic agents ketamine, etomidate, thiopental, and propofol on seizure parameters and seizure quality in electroconvulsive therapy: A retrospective study. European Archives of Psychiatry and Clinical Neuroscience, 264, 255-261.

Jacob, P., Gogi, P.K.V., Srinath, S., Thirthalli, J., Girimaji, S., Seshadri, S. et al. (2014.). Review of electroconvulsive therapy practice from a tertiary Child and Adolescent Psychiatry Centre. Asian Journal of Psychiatry, 12, 95-99.

Janke, C., Bumb, J. M., Aksay, S. S., Thiel, M., Kranaster, L. \& Sartorius, A. (2015). Ketamin als Anästhetikum bei der Elektrokrampftherapie. Anaesthesist, 64, 357-364.

Karayağmurlu, A., Coșkun, M., Elboğa, G., Ghazziudin, N., Karayağmurlu, E. Gögçen, C. et al. (2020). Efficacy and safety of electroconvulsive therapy in adolescents: A retrospective chart review study from Turkey. Journal of ECT, 36, 54-59.

Kho, K.H., van Vreeswijk, M.F., Simpson, S. \& Zwinderman, A.H. (2003). A meta-analysis of electroconvulsive therapy efficacy in depression. Journal of ECT, 19,139-147.

Lally, J., Tully, J., Robertson, D., Stubbs, B., Gaughran, F. \& MacCabe, J.H. (2016). Augmentation of clozapine with electroconvulsive therapy in treatment resistant schizophrenia: A systematic review and meta-analysis. Schizophrenia Research, 171, 215-224.

Leiknes, K.A., Jarosh-von Schweder, L. \& Høie, B. (2012). Contemporary use and practice of electroconvulsive therapy worldwide. Brain and Behavior, 2, 283-344.

Loh, N., Nickl-Jockschat, T., Sheldrick, A.J. \& Grözinger, M. (2013). Accessibility, standards and challenges of electroconvulsive therapy in Western industrialized countries: A German example. World Journal of Biological Psychiatry, 14, 432-440.

Loiseau, A., Harrisson, M.-C., Beaudry, V. \& Patry, S. (2017). Electroconvulsive therapy use in youth in the province of Quebec. Journal of the Canadian Academy of Child and Adolescent Psychiatry, 26, 4-11.

Loo, C.K., Mahon, M., Katalinic, N., Lyndon, B. \& Hadzi-Pavlovic, D. (2011). Predictors of response to ultrabrief right unilateral electroconvulsive therapy. Journal of Affective Disorders, 130, 192-197.

McCall, W.V., Foreword, In N.W. Ghaziuddin \& G. Walter (Eds.), (2013), Electroconvulsive therapy in children and adolescents, New York: Oxford University Press.

Mitchell, S., Hassan, E. \& Ghaziuddin, N. (2018). A follow-up study of electroconvulsive therapy in children and adolescents. Journal of ECT, 34, 40-44.

Mulder, L.A.C. \& Grootens, K. P. (2020). The incidence of post-electroconvulsive therapy headache: A systematic review. Journal of ECT, 36, e22-e28.

Nordanskog, P., Hultén, M., Landén, M., Lundberg, J., von Knorring, L. \& Nordenskjöld, A. (2015). Electroconvulsive therapy in Sweden 2013: Data from the national quality register for ECT. Journal of ECT, 31, 263-267.

Pagnin, D., de Queiroz, V., Pini, S. \& Cassano, G.B. (2004). Efficacy of ECT in depression: A meta-analytic review. Journal of ECT, 20, 13-20. 
Paillère-Martinot, M.L., Zivi, A. \& Basquin, M. (1990). [Use of electroconvulsive therapy in adolescence]. Encéphale, 16, 399-404.

Popiolek, K., Bejerot, S., Brus, O., Hammar, Å., Landén, M., Lundberg, J. et al. (2019). Electroconvulsive therapy in bipolar depression - effectiveness and prognostic factors. Acta Psychiatrica Scandinavica, 140, 196-204.

Puffer, C.C., Wall, C.A., Huxsahl, J.E. \& Frye, M.A. (2016). A 20 year practice review of electroconvulsive therapy for adolescents. Journal of Child and Adolescent Psychopharmacology, 26, 632-636.

Reinhardt, M., Wunsch, K., Weirich, S., Häßler, F. \& Buchmann, J. (2018). Fallbericht einer 15-jährigen Patientin mit einer schweren, wahnhaften Depression, behandelt mit Elektrokonvulsionstherapie. Zeitschrift für Kinder- und Jugendpsychiatrie und Psychotherapie, 46, 536-541.

Rootes-Murdy, K., Carlucci, M., Tibbs, M., Wachtel, L.E., Sherman, M. F., Zandi, P.P. et al. (2019). Non-suicidal self-injury and electroconvulsive therapy: Outcomes in adolescent and young adult populations. Journal of Affective Disorders, 250, 94-98.

Sackeim, H.A., Ross, F.R., Hopkins, N., Calev, L. \& Devanand, D.P. (1987). Subjective side effects acutely following ECT: Associations with treatment modality and clinical response. Convulsive Therapy, 3, 100-110.

Sartorius, A., Aksay, S.S., Bumb, J.M., Janke, C. \& Kranaster, L. (2015). Psychomimetic adverse effects of S-ketamine as an anesthetic for electroconvulsive therapy are related to low doses and not to axis I diagnosis. Journal of ECT, 31, 73-74.

Sartorius, A., Pycha, R., Grözinger, M. \& Conca, A. (2013). Praktische Durchführung der EKT, In M. Grözinger, A. Conca, T. NicklJockschat \& J. Di Pauli (Hrsg.)., Elektrokonvulsionstherapie kompakt (S. 109-125). Berlin: Springer.

Semkovska, M., Landau, S., Dunne, R., Kolshus, E., Kavanagh, A., Jelovac, A. et al. (2016). Bitemporal versus high-dose unilateral twice-weekly electroconvulsive therapy for depression (EFFECT-Dep): A pragmatic, randomized, non-inferiority trial. American Journal of Psychiatry, 173, 408-417.

Semkovska, M. \& McLoughlin, D.M. (2010). Objective cognitive performance associated with electroconvulsive therapy for depression: A systematic review and meta-analysis. Biological Psychiatry, 68, 568-577.

Shoirah, H. \& Hamoda, H.M. (2011). Electroconvulsive therapy in children and adolescents. Expert Review of Neurotherapeutics, 11,127-137.

Stein, D., Kurtsman, L., Stier, S., Remnik, Y., Meged, S. \& Weizman, A. (2004). Electroconvulsive therapy in adolescent and adult psychiatric inpatients: A retrospective chart design. Journal of Affective Disorders, 82, 335-342.
Thompson, J.W. \& Blaine, J.D. (1987). Use of ECT in the United States in 1975 and 1980. American Journal of Psychiatry, 144, 557-562.

Walter, G., Koster, K. \& Rey, J. M. (1999). Electroconvulsive therapy in adolescents: Experience, knowledge, and attitudes of recipients. Journal of the American Academy of Child \& Adolescent Psychiatry, 38, 594-599.

Wang, G., Zheng, W., Li, X.-B., Wang, S.-B., Cai, D.-B., Yang, X.-H. et al. (2018). ECT augmentation of clozapine for clozapine-resistant schizophrenia: A meta-analysis of randomized controlled trials. Journal of Psychiatric Research, 105, 23-32.

Wang, S., Yang, C., Jia, J., Zhou, Y. \& Zheng, Y. (2018). Use of electroconvulsive therapy in adolescents with schizophrenia in China. Child and Adolescent Psychiatry and Mental Health, 12, 49.

Wang, Z.-M., Zhu, H., Pan, Y.-L., Chiu, H. F.K., Correll, D. U., Ungvari, G.S. et al. (2015). Electroconvulsive therapy and its association with demographic and clinical characteristics in Chinese psychiatric patients. Journal of ECT, 31, 114-118.

Weirich, S., Reinhardt, M., Buchmann, J., Dück, A., Höppner, J., Hofmockel, R. et al. (2020). Elektrokonvulsionstherapie - bei Kindern und Jugendlichen mit Schizophrenie gut verträglich. Fortschritte der Neurologie · Psychiatrie, 88, 495-499.

Zhand, N., Courtney, D.B. \& Flament, M.F. (2015). Use of electroconvulsive therapy in adolescents with treatment-resistant depressive disorders: A case series. Journal of ECT, 31, 238-245.

\section{Historie}

Manuskript eingereicht: 20.05.2020

Nach Revision angenommen: 28.01.2021

Onlineveröffentlichung: 15.03.2021

\section{Interessenkonflikt}

Die Autoren haben keinen Interessenkonflikt.

\section{Förderung}

Open Access-Veröffentlichung ermöglicht durch die Universitätsmedizin Göttingen.

\section{Isabel Methfessel}

Universitätsmedizin Göttingen

Klinik für Psychiatrie und Psychotherapie

Von-Siebold-Str. 5

37075 Göttingen

Deutschland

Isabel.methfessel@med.uni-goettingen.de 